\title{
Tunable particle separation in a hybrid dielectrophoresis (DEP)- inertial microfluidic device
}

Jun Zhang ${ }^{\mathrm{a}+}$, Dan Yuan ${ }^{\mathrm{b}}$, Qianbin Zhao ${ }^{\mathrm{b}+}$, Sheng Yan ${ }^{\mathrm{b}}$, Shi-Yang Tang, Say Hwa Tan ${ }^{\mathrm{c}}$, Jinhong Guo $^{\mathrm{d}}$, Huanming Xia ${ }^{\mathrm{a}}$, Nam-Trung Nguyen ${ }^{*}$, Weihua $\mathrm{Li}^{*}{ }^{*}$

${ }^{a}$ School of Mechanical Engineering, Nanjing University of Science and Technology, Nanjing 210094, China

${ }^{\mathrm{b}}$ School of Mechanical, Materials and Mechatronic Engineering, University of Wollongong, Wollongong, NSW 2522, Australia. E-mail: weihuali@uow.edu.au; Fax: +61 24221 3238; Tel: +61242213490

${ }^{\mathrm{c}}$ Queensland Micro and Nanotechnology Centre, Griffith University, Brisbane, QLD 4111, Australia. E-mail: nam-trung.nguyen@griffith.edu.au; Fax: +61 07373 58021; Tel: +61 (07) 373 53921

${ }^{\mathrm{d}}$ School of Electronic Engineering, University of Electronic Science and Technology of China, No. 2006, Xiyuan Ave, Chengdu 611731, China

* corresponds to corresponding authors; ${ }^{+}$corresponds to equal contribution

\section{Highlights}

- An innovative hybrid dielectrophoresis (DEP)-inertial microfluidic devices for particle separation is proposed;

- Dielectrophoresis (DEP) force can tune the inertial particle focusing in real-time in the DEPinertial microfluidic platform;

- Separation resolution and cut-off size of particle mixture can be adjusted in the DEP-inertial microfluidic platform without the need of redesigning microchannel structure or dimension.

\section{Abstract}


Particle separation is indispensable in many microfluidic systems and holds a broad range of biomedical applications. Inertial microfluidic devices that work solely on intrinsic hydrodynamic forces and inertial effects can offer label-free, high throughput and high efficiency separation performance. However, the working range of the current inertial microfluidic systems is obtained by tailoring the inertial lift forces and secondary flow drag through flow speed. Each channel design is normally effective for specific target particles, which inevitably lacks the flexibility for various particle mixtures. Redesigning the structure and dimension of microchannels for new sets of particle mixtures is often time-consuming and expensive. In this work, by introducing an external dielectrophoretic force field and coupling it with inertial forces, we proposed here an innovative hybrid DEP-inertial microfluidic platform for particle tunable separation. The working principle of the device was explained and its functionality was validated by experiments. In addition, the dimension of target particle mixture can be varied by adjusting the electrical voltage without redesigning the channel structure or dimensions. It is expected that the proposed DEP-inertial concept can work as a flexible platform for a wide range of biomedical applications.

Key words: Hybrid microfluidics, inertial microfluidics, dielectrophoresis (DEP), particle separation

\section{Introduction}

Particle separation is indispensable for a wide range of basic biological, chemical and clinical studies [1, 2]. The most commonly used technology for biological sample separation is centrifugation. However, it is limited by the bulky size, high cost, and intensive labor [3, 4]. Furthermore, it can be dangerous if improperly operated because the system contains high-speed rotating parts. Another common technique is based on membrane filter that uses the pore size to selectively transport or block target particles through the membrane. However, this approach may suffer from the severe clogging and fouling problems [5].

Microfluidic technology has emerged as a promising solution for particle separation. The technology manipulates and separates particles by well-defined microstructures and precisely controlled physical fields at the micrometer scale. Microfluidics is a simpler, cheaper, and more efficient technique compared to the conventional macroscale technology. In microfluidics, 
particle manipulation and separation can be classified as active and passive according to the origin of dominant manipulating forces. Active techniques employ active external force fields [69], while passive microfluidic devices depend on the intrinsic hydrodynamic forces and microchannel structure [10-12]. Generally, active microfluidics can precisely control particles of interest and adjust their position in real time. However, the flow speed is slow because a long residual time is required to exert sufficient external manipulating force to the target particles, thus limiting the overall throughput. In contrast, passive microfluidics has the advantages of simple structure, robustness, and high throughput.

Inertial microfluidics is an unconventional passive microfluidic technology, that manipulates and separates microparticles by the finite inertial effects of fluid [12]. In contrast to the conventional microfluidic technologies in which flow speed is very low and inertial effects are negligible, inertial microfluidics works at a much higher flow speed where fluid inertia cannot be neglected. Due to the superior advantages of high throughput, precise manipulation, simplicity, and robustness, inertial microfluidic technology has received extensive attention and has developed rapidly within the last two decades [12-14]. A variety of microchannel structures have been developed for particle inertial focusing and separation, including straight $[15,16]$, spiral [17-19], serpentine [20, 21], straight channel with expansion-contractions [22-25] and with pillar arrays [26, 27]. Meanwhile, various applications using the inertial microfluidic technique have been explored such as extraction of blood plasma [28], separation of particles and cells [29, 30], solution exchange [31, 32], cell enrichment [33], isolation of circulating tumour cells (CTCs) [34-36], detection of malaria pathogen [37], microfiber fabrication [38], cell cycle synchronization [39], cell encapsulation [40], and stretching of single cell [41] etc. However, once the microfluidic devices are designed and fabricated, their applicable range of the target particles is normally restricted. Therefore, this technology lacks the flexibility and tunability to adapt to different particle samples once the microchannel structure and dimensions are fixed.

Hybrid techniques, which combine both active and passive methods, have been proposed to overcome their individual shortcomings and integrate the benefits from both methods [42]. The reported hybrid techniques include combination of dielectrophoresis (DEP) with deterministic lateral displacement (DLD) [43], hydrophoresis [44] or field flow fractionation (FFF) [45], magnetophoresis (MP) with hydrodynamics [46] or viscoelastic focusing [47, 48], virtual DLD with acoustophoresis (AP) [49] et al. However, very few attempts to combine inertial 
microfluidics and active techniques have been reported [36, 50-52]. Moon et al. [52] proposed a novel platform which combines multi-orifice flow fractionation (MOFF) and DEP techniques to separate human breast cancer cells (MCF-7) from spiked blood samples. The inertial separation by MOFF takes advantage of high-throughput filtration of normal blood cells, and the serially connected DEP separator plays as a precise post-processor to further enhance the efficiency of separation. Later, Toner et al. successfully developed an inertial focusing-enhanced microfluidic CTC capture platform (CTC-iChip) for unbiased, rapid, and automated isolation of CTCs [36, 50, 53]. The CTC-iChip take advantages of techniques of DLD, inertial focusing and MP. In the platform, the DLD firstly removes red blood cells (RBCs) from the raw blood sample. The remaining white blood cells (WBCs) and CTCs enter an asymmetric serpentine microchannel and become promptly focused on a single path by the inertial forces. Finally, at the end of the serpentine channel, a strong magnetic field is applied to deflect target WBCs or CTCs that are magnetically labeled, so that CTCs can be isolated effectively. Very recently, Zhou et al. [51] demonstrated a hybrid microfluidic technique that combines passive inertial focusing with active negative magnetophoresis to separate diamagnetic particles in ferrofluid. However, in these works, each functioning unit was connected in a serial manner, and the physical fields of functional units are not fully coupled. Therefore, the connected units were not to optimize the separation performance of inertial microfluidics, but working as an additional post-processing step.

Our previous work explored the feasibility of fully coupling dielectrophoretic (DEP) forces and inertial forces in serpentine microchannels, and DEP force was successfully demonstrated to modify the particle inertial focusing patterns [54]. In the present work, we further modified the DEP-inertial microfluidic platform and applied it for particle separation. First, the separation mechanism of the proposed platform was explained. Then, through the elimination of top inertial focusing positions by a sheath flow, the focusing positions of all particles were pinched close to the channel bottom where microelectrodes are patterned, so that the DEP force can modify all the particles' focusing positions. Subsequently, the effects of electric voltage on the particle inertial focusing patterns of differently-sized particles were investigated. Finally, successful separation of binary particle mixture was demonstrated experimentally. Since the sizes of target binary particle mixture can be tuned by adjusting electric voltage without requirement of redesigning 
the structure or dimension of microchannels, the proposed novel platform provides more flexibility over the conventional inertial microfluidic devices.

\section{Theory}

\subsection{Inertial migration}

When particles are dispersed in tubular flow with a finite inertia, inertial migration phenomenon that randomly distributed particles migrate laterally toward several cross-sectional equilibrium positions will happen. The phenomenon is widely recognised by the counteraction of two inertial lift forces: the shear gradient lift force $F_{L S}$, and the wall lift force $F_{L W}$. The shear gradient lift force $F_{L S}$ originates from the curvature of the fluid velocity parabolic profile and its interaction with a finite size particle, while the wall lift force $F_{L W}$ is a result of the disturbance of the flow field around the suspending particles and its reflection off the wall [12, 13]. The equilibrium positions correspond to the location where the resultant of the two inertial lift forces is zero. The net inertial lift force $F_{L}$ can be expressed as follows when the particle size is small compared to the channel size [12]:

$$
\begin{gathered}
F_{L}=\frac{\rho_{f} U_{m}^{2} a^{4}}{D_{h}^{2}} f_{L}\left(R_{C}, z\right) \\
R_{C}=\frac{\rho_{f} U_{m} D_{h}}{\mu_{f}}
\end{gathered}
$$

where $\rho_{f}, U_{m}$ and $\mu_{f}$ are fluid density, maximum velocity, and dynamic viscosity, respectively; $a$ is particle diameter, and $D_{h}$ is the hydraulic diameter of the channel. $f_{L}\left(R_{C}, z\right)$ is the lift coefficient of the net inertial lift force, which is a function of the position of particles within the cross-section of channel $z$ and channel Reynolds number $R_{C}$ [12]. The locations where $f_{L}=0$ correspond to the dynamic equilibrium positions for particles in the channel cross-section.

The introduction of channel curvature often induces counter-rotating secondary flows (or Dean flow) which impose an additional drag force on the particles to modify and assist particle inertial focusing progress [21]. In serpentine microchannels, the direction of secondary flow is alternated along the channel length, which results in alternating secondary flows. In the symmetric serpentine channels, the focusing pattern (e.g. position and number of dynamic equilibrium positions) is always determined by the balance between secondary flow drag and 
inertial lift force. If the secondary flow drag is larger than the inertial lift force, particles focus at the center of the microchannel. Otherwise, if the secondary flow is too weak to alter the focusing pattern dominated by the inertial lift force, and particles are normally ordered along the two sidewalls [20].

\subsection{Dielectrophoresis}

Dielectrophoretic (DEP) force is a result of the interaction of non-uniform electrical field and field-induced electrical polarisation of particles. A time-averaged DEP force exerts on particles when they are immersed in an alternating electrical field [49]:

$$
F_{D E P}=2 \pi \varepsilon_{m} r^{3} \operatorname{Re}[K(\omega)] \nabla\left|E_{m s}\right|^{2}
$$

where $\varepsilon_{m}$ is the permittivity of the medium, $r$ represents the particle radius, $\left|E_{r m s}\right|$ is the rootmean-squared value of the applied electrical field, and $K(\omega)$ refers to the Clausius-Mossotti $(\mathrm{CM})$ factor. Re indicates the real part of the factor, and $K(\omega)\left(=\left(\varepsilon_{p}^{*}-\varepsilon_{m}^{*}\right) /\left(\varepsilon_{p}^{*}+2 \varepsilon_{m}^{*}\right)\right)$ depends on the relative permittivity of the particle and the suspending medium, as well as the frequency of the external electric field. $\varepsilon^{*}=\varepsilon-\mathrm{i} \sigma / \omega$ is the complex permittivity, $\sigma$ is the electrical conductivity, and $\omega$ is the angular frequency of the electric field. The subscripts $p$ and $m$ denote the particle and suspending medium, respectively.

If $\operatorname{Re}[K(\omega)]>0$, the DEP is positive and particles are attracted into the regions of strong electric fields, while if $\operatorname{Re}[K(\omega)]<0$, the DEP force is negative and particles are repelled from those regions.

In the present work, an interdigitated electrode (IDE) array was patterned on the bottom of the microchannel to generate a negative DEP (n-DEP) on the particles and push them upwards, Figure 1(a, b). Since the DEP force in the horizontal and lateral direction is too weak to alter particle positions due to a high flow speed, the vertical DEP force $\left(F_{D E P z}\right)$ counteracts with the inertial lift force along the vertical direction and adjusts the dynamic equilibrium positions of particles. Without a specific notice, the DEP force in the whole manuscript indicates the DEP force component in the vertical $(z)$ direction.

\subsection{Particle separation mechanism in DEP-inertial microfluidic platform}


In the previous work, we demonstrated that inertial focusing pattern could be modified by coupling a dielectrophoresis force [54]. However, the micro-electrodes were patterned on the bottom of microchannel and the generated DEP force could only be effective within the bottom region of microchannel. This is because the magnitude of DEP force decreased sharply along the vertical direction $(z)$. The particles within the top region of microchannel cannot be modified simultaneously, which hinders particle separation using DEP modification. Therefore, in this work, we proposed to eliminate the top inertial equilibrium positions by a top sheath flow, Figure 1(a). The top sheath flow spreads downward and pushes all the particles into the bottom region of microchannel at the inlet, so that all particles are focused on the bottom region of the microchannel.

Then, after an alternating electrical field is applied to generate the DEP force on the particles, the DEP force $F_{D E P}$ competes with the inertial lift force $F_{L}$ along the vertical direction (z), levitating particles upward where the magnitude of secondary flow becomes stronger, Figure 1(c). The net effect of the stronger secondary flow is that particles migrate toward the channel centerline along the lateral direction. Since different scaling orders of DEP force and inertial lift force on particle size, the vertical equilibrium position of particles is highly dependent on particle size, subsequently causing differential focusing positions of particles along the lateral direction $(y)$.

Here, a dimensionless factor $\delta$ was defined as the magnitude ratio of DEP force to inertial lift force:

$$
\delta=\left|\frac{F_{D E P}}{F_{L}}\right|_{C}=\frac{\pi D_{h}^{2} \varepsilon_{m} \operatorname{Re}[K(\omega)] \nabla\left|E_{r m s}\right|^{2}}{4 \mid f_{L}\left(R_{C}, Z\right) a \rho_{f} U_{m}^{2}} \propto a
$$

The recent work of Zhou and Papautsky [55] indicates that $f_{\mathrm{L}}$ is proportional to $a^{-2}: f_{L} \propto$ $a^{-2}$. Therefore, $\delta$ is proportional to particle diameter $\delta \propto a$. That means, particles with a larger size will be levitated upwards higher than that of smaller ones under the same conditions, and they are focused closer to the channel centerline than the smaller particles. A more detailed understanding is provided in the supplementary information. Furthermore, a tiny location difference along the vertical direction $(z)$ can be amplified along the lateral direction $(y)$, which facilitates high efficient particle separation. This is the basic separation principle of the proposed DEP-inertial microfluidic platform, Figure 1(b).

\section{Materials and methods}




\subsection{Design and fabrication of DEP-inertial microfluidic device}

The microfluidic channel used in our experiments consisted of 15 symmetric zigzag periods. The width of the microchannel was $200 \mu \mathrm{m}$. The length and width of each U-turn were both $700 \mu \mathrm{m}$. The height of the channel was $40 \mu \mathrm{m}$. The polydimethylsiloxane (PDMS) microfluidic channel was fabricated by the standard photolithography and soft lithography techniques [56]. In addition to the inlet and outlet holes, which are used for infusion and collection of particle samples, a fluidic hole on the PDMS slab for sheath flow was also formed delicately using a custom-made punch. For the glass slide with microelectrodes, a 50-nm Ti/150-nm Pt electrode layer was patterned using the standard lift-off process [57]. The gap and width of the interdigitated electrodes (IDEs) were both $20 \mu \mathrm{m}$, and the total length of IDEs was $13.5 \mathrm{~mm}$. In order to form a closed microchannel, both the PDMS slab and the glass slide were exposed to an oxygen plasma for 3 mins, and they were permanently bonded after being carefully aligned and contacted. A photograph of the fabricated device was shown in Figure 1(d).

\subsection{Particle preparation}

Fluorescence-labelled polystyrene particles were purchased from Thermo Fisher Scientific. The average diameters of fluorescent particles are $4.8 \mu \mathrm{m}, 8 \mu \mathrm{m}$ and $13 \mu \mathrm{m}$ respectively. They were suspended in deionized (DI) water with a weight ratio about 0.025\%. Tween 20 (SIGMAALDRICH, Product No. P9416) with a weight ratio of $0.1 \%$ was added to prevent particles from aggregation. To conduct separation tests, binary polystyrene beads mixtures $(5 \mu \mathrm{m}$ and $13 \mu \mathrm{m}, 8$ $\mu \mathrm{m}$ and $13 \mu \mathrm{m}$, respectively) were prepared in the DI water with Tween 20 . The bulk conductivity and permittivity of the polystyrene beads are $2.4 \times 10^{-4} \mathrm{~S} / \mathrm{m}$ and $2.6 \varepsilon_{0}$, respectively. The conductivity and permittivity of DI water are $1.5 \times 10^{-4} \mathrm{~S} / \mathrm{m}$ and $78 \varepsilon_{0}$, respectively [58].

\subsection{Experimental setup and methods}

The microfluidic device was placed on an inverted microscope (CKX41, Olympus, Japan), illuminated by a mercury arc lamp. The particle suspension and sheath flow were infused into the microchannel with specific flow rates by two syringe pumps (Legato 100, KD Scientific). A sinusoidal signal with a frequency of $1 \mathrm{MHz}$ was generated by a waveform generator (33250A, Agilent, USA) and amplified by an RF power amplifier (TIA-1000-1R8-2, Mini-Circuits, USA). 
Copper wires with one end soldered onto the IDEs pads were connected to the power amplifier to activate the electrodes. The dielectrophoresis (DEP) force was induced by the sinusoidal voltage and its magnitude can be controlled by the applied voltage magnitude. The DEP force competes with inertial lift force along the vertical direction and adjusts particles' focusing positions in the cross-section. Fluorescent images of particle trajectories were observed and captured by a CCD camera (Optimos, Q-imaging, Australia), and then post-processed and analysed using Q-Capture Pro 7 (Q-imaging, Australia) software. The concentrations of particles were measured by a hemocytometry. The purity of particle suspensions collected from different outlets was calculated from three measurements by a hemocytometry.

\section{Results and discussions}

\subsection{Elimination of top inertial focusing streaks by a sheath flow}

Inertial focusing in the symmetric serpentine channel comprises of three different focusing patterns at various flow rates: (1) two-sided inertial focusing pattern; (2) transitional inertial focusing pattern; and (3) single central inertial focusing pattern [20]. The two-sided inertial focusing pattern was chosen in this work and the total flow rate is fixed at $300 \mu \mathrm{l} / \mathrm{min}$. As we know, there are symmetric focused streaks on the top and bottom of microchannel along the channel vertical center plane, and the DEP force exerted by the bottom patterned IDEs electrodes can only be effective for the bottom focused streaks. Therefore, in order to eliminate the focused streaks on the top half of microchannel, a top sheath flow was implemented. In order to evaluate the effectiveness of top sheath flow on the elimination of top focused streaks, a PDMS microfluidic device was fabricated from three PDMS slabs using the method reported by Guan et al. [59] to facilitate the observation of the side view. When the flow rate ratio of sheath flow to sample flow was zero (no sheath flow), Figure 2(i), two focused streaks along the top and bottom of channel appeared, Figure 2(ii-iv) and Movie S1. Within our expectation, when the flow rate ratio of sheath flow to sample flow was set as 2:1, all particles were pressed down to the bottom of microchannel at the inlet, Figure 2(v), and there was only one focused streak along the bottom of microchannel, Figure 2(vi-ix) and Movie S2. In addition, we varied the flow rate ratio of sheath flow to sample flow and found that if the ratio was not less than 2:1, most particles could be pinched and focused within the bottom half of channel. Therefore, in the following test, we 
fixed the flow rate ratio of sheath flow to sample flow at 2:1, so that all particles can be focused within the channel bottom, where DEP force can effectively be exerted on them.

\subsection{Modification of inertial focusing positions by n-DEP force}

After successful elimination of top focusing streaks by the top sheath flow, we investigated the effects of electrical voltages on the focusing patterns (positions and numbers) of particles on the bottom of the microchannel. Figure 3(a) and Movie S3 show the varying particle focusing streak patterns under an increasing electrical voltage in the DEP-inertial microfluidic device. When the applied electric voltage $V_{p p}=0 \mathrm{v}$, there was no DEP force exerted on 8- $\mu \mathrm{m}$ particles, particles were focused along the two sidewalls, Figure $3 \mathrm{a}(\mathrm{i})$. Increasing the applied voltage $V_{p p}$, the DEP force emerged to levitate particles upwards, and focused particle streaks shifted gradually towards the channel centerline from the top-down view, Figure $3 \mathrm{a}(\mathrm{ii}-\mathrm{v})$. Above a threshold of $V_{p p}$, two focused streaks combined as a single one, Figure $3 \mathrm{a}(\mathrm{vi})$, and the streak width shrank with the increment of $V_{p p}$, Figure $3 \mathrm{a}$ (vii). Continuing to raise the applied voltage, particles began to escape from the central focused streak towards the two sidewalls, Figure $3 a$ (viii), and the portion of the escaped particles grew significantly with increment of $V_{p p}$, Figure $3 \mathrm{a}(\mathrm{ix})$, and finally all the particles moved toward the two sidewalls, Figure $3 a(x)$. The fluorescence profiles of the focused streaks at the end of serpentine channel clearly indicate the transformation process of the particle inertial focusing with an increasing voltage in the DEP-inertial microfluidic device, Figure $3 b$.

To further deepen the understanding of inertial focusing position transformation process, we analyzed the inertial focusing positions of particles within channel cross-sections under the interaction of DEP force and inertial lift force along the vertical direction, as shown in Figure 4. Five typical focusing patterns under an increasing applied electric voltage were shown in Figure 4a, with the corresponding speculated cross-sectional dynamic equilibrium positions of particles depicted in Figure 4b. Under an increasing electric voltage, (i) particles are initially focused along two sidewalls; (ii) two-sided focused streaks migrate toward channel centre; (iii) two focused streaks merge as a single focused streak around the channel centreline; (iv) two additional focused streaks appear along two sidewalls besides the central focused streak; finally (v) the central focused streak vanishes with two-sided streaks remained. 
From the aspects of dynamics, there are three different force balance stages according to the counteraction of DEP force and inertial lift force along the vertical direction, Figure 4c. (I) At a relatively low electric voltage, there are two stable equilibrium positions A and D (green square symbols) along the vertical direction $(z)$ where the net force $\left(F_{D E P}+F_{L z}\right)$ on both sides is pointing to themselves, Figure 4c(i). In this work, as we demonstrated above, by applying a top sheath flow, all the particles at the inlet were pushed downward and maintained within the bottom of microchannel, therefore all the particles are focused at point A and no particles are located at point $\mathrm{D}$. Point $\mathrm{C}$ is an unstable position where the net force on both sides directs outwards, and particles can easily escape towards both sides by a weak disturbance. Within this stage, twosided focused streaks of particles shift toward channel centerline and merge as a single tight focused streak with the vertical ascent of particles, and this stage includes the inertial focusing patterns (i-iii) in Figure 4(a, b); (II) Increasing electrical voltage, point A moves upwards and point $\mathrm{C}$ moves downwards, and eventually they contact and become the point B, Figure 4c(ii). Point B is a saddle position, where the net force on both sides is directing upward. A small disturbance to shift particles below point $\mathrm{B}\left(\mathrm{z}<\mathrm{z}_{\mathrm{B}}\right)$ could bring back particles to the location $\mathrm{B}$ due to the upward net force $\left(F_{D E P}+F_{L z}\right)$, however, a slight disturbance above point $\mathrm{B}\left(\mathrm{z}>\mathrm{ZB}_{\mathrm{B}}\right)$ could never bring particles back. In the experiments, a portion of particles on point B escaped and moved up to stable point $\mathrm{D}$ and the remaining particles were staying at point $\mathrm{B}$. Therefore, as we observed experimentally, there were three focused streaks from the top-down view, corresponding to focusing pattern (iv) in Figure 4(a); (iii) When the applied voltage exceeds a threshold, the net force $\left(F_{D E P}+F_{L z}\right)$ is larger than zero and directing upward throughout the bottom half region, thus all the particles will migrate upward to point $\mathrm{D}$, which corresponds to focusing pattern (v) in Figure 4(a, b).

Besides the electric voltage, the electric frequency is another important parameter in DEP manipulation, and its influence on particle focusing positions in DEP-inertial microfluidic platform was investigated. As shown in Figure 5(a), particles are focused as two sided streaks near the sidewalls at a low electric frequency $(f=0.1 \mathrm{MHZ})$, and the focusing streaks shift toward the channel center when increasing the electric frequency, and finally they emerged as a single central focusing streak $(f=1 \mathrm{MHZ})$. The reason behind the phenomenon can be explained by the $\operatorname{Re}[K(\omega)]$ which determines the magnitude of DEP force under different electric frequencies, Figure 5(b). The magnitude of $\operatorname{Re}[\mathrm{K}(\omega)]$ is much smaller $(\sim 0.199)$ at a low frequency $(0.1 \mathrm{MHZ})$ 
than that at the frequency of $1 \mathrm{MHZ}$, which means that DEP force is not strong enough to levitate particles and particles can only focus as two sided streaks. The calculation of $\operatorname{Re}[\mathrm{K}(\omega)]$ can be found in the supplementary information.

\subsection{Effects of particle size}

Particle size is an important parameter for particle separation in microfluidics, thus the effects of particle size on the focusing phenomena of the proposed DEP-inertial microfluidic devices were also investigated. Besides 8- $\mu \mathrm{m}$ particles, 5- $\mu \mathrm{m}$ and 13- $\mu \mathrm{m}$ particles suspensions were prepared in DI water and infused into the microfluidic device with the same flow rate ratio of sample flow to sheath flow, respectively. By increasing the applied voltage, we found that the inertial focusing patterns of $5-\mu \mathrm{m}$ and 13- $\mu \mathrm{m}$ transformed with a similar trend with that of $8-\mu \mathrm{m}$ particles, as shown in the Figures S1 and S2, that is the two-sided focused streaks moved toward the channel centerline and merged as a single one, then it was split as three focused streaks, and finally returned back to the two-sided focused streaks.

A particle diameter - electrical voltage map to illustrate the working regions of different particle focusing patterns was depicted in Figure 6, which can help to determine the suitable electrical voltage for particle separation. The map divided particle focusing patterns into three basic regions. In region I, particles are focused along two sidewalls due to the weak DEP force; In region II, a sufficient DEP force levitates particles so that particles can be pinched as a single focused streak at the channel center; In region III, DEP force is too strong and the resultant of DEP force and inertial lift force cannot create stable position within the bottom area of channel, thus a portion of and even all particles escape from the centrally focused streak and migrate to the two sidewalls. It should be noted that the regions I and II in the map (Figure 6) correspond to the stage I in the dynamic analysis (Figure 4c), and region III in the map (Figure 6) includes the stages II and III in the dynamic analysis (Figure 4c). From the map, it is obvious that the critical electric voltages for the conversion of these three regions are highly dependent on particle size. For example, the minimum electric voltages $\mathrm{V}_{\mathrm{C} 1}$ for the formation of the centrally focused streak (region II) for $5-\mu \mathrm{m}, 8-\mu \mathrm{m}$ and $13-\mu \mathrm{m}$ particles are $48 \mathrm{v}, 39 \mathrm{v}$ and $27 \mathrm{v}$, respectively. Therefore, by selecting a suitable electric voltage at which binary particles are located in different regions in 
the map, e.g. one is in region I and the other is in region II, then it is possible to conduct effective particle separation in the proposed DEP-inertial microfluidic device, as we will demonstrate in the following.

\subsection{Particle separation}

In inertial microfluidics, channel structures (e.g., channel curvature [18, 19, 34, 39], obstacle array [22, 60], cross-sectional dimension [15] and cross-sectional shape [16, 30, 35]) are often manipulated to enable differential focusing positions of particles or distinct migration speed to achieve effective separation. In these works, the input flow rate is the only parameter that can be adjusted in the separation process. In this work, through the novel hybrid DEP-inertial microfluidic platform, we demonstrated that an additional parameter electric voltage can be adjusted in real-time to facilitate particle separation. Figure 7a shows the focusing positions and focusing widths of $5-\mu \mathrm{m}$ and $13-\mu \mathrm{m}$ particles under varying electric voltages, so that the lateral position difference of binary particle streaks can be easily determined. When the applied electric voltage was zero, no DEP force was exerted on the particles, and both $13-\mu \mathrm{m}$ and $5-\mu \mathrm{m}$ particles were focused along the two sidewalls with focusing bands overlapped each other, Figure 7b, therefore, it is impossible to achieve particle separation at this situation. By adjusting the electrical voltage to $\mathrm{V}_{\mathrm{pp}}=27 \mathrm{v}$, the small $5-\mu \mathrm{m}$ particles are still focused along two sidewalls while $13-\mu \mathrm{m}$ particles have combined as a single focused streak along channel centerline, Figure 7c. The lateral distance of particle streaks can be as large as $29 \mu \mathrm{m}$. Therefore, the binary particle mixture can be separated by collecting the differential focused streaks at the end of the microchannel.

In order to evaluate separation performance of the DEP-inertial microfluidic device, we prepared a binary particle mixture $\left(13-\mu \mathrm{m}\right.$ and $5-\mu \mathrm{m}$ in diameter), with concentrations of $8.5 \times 10^{5}$ counts $/ \mathrm{ml}$ for $13-\mu \mathrm{m}$ and $1.4 \times 10^{6}$ counts $/ \mathrm{ml}$ for $5-\mu \mathrm{m}$. Infusing the particle mixture into the DEP-inertial microfluidic device and setting the applied electric voltage as $27 \mathrm{v}$, the particle suspensions after processing were collected respectively from the two-sided outlets and the central outlet. The concentrations of particle suspension before and after processing were measured by a hemocytometer, the fluorescence images of binary particle mixture from the inlet and two outlets were shown in Figure 8a. The particle purity was increased from $38 \%$ to $93.8 \%$ 
for $13-\mu \mathrm{m}$ particles, and $62 \%$ to $100 \%$ for $5-\mu \mathrm{m}$ particles, Figure $8 \mathrm{~b}$. Meanwhile, the separation efficiency of $13-\mu \mathrm{m}$ and $5-\mu \mathrm{m}$ particles are about $100 \%$ and $96 \%$ respectively, Figure 8c.

Besides the binary particles $(13-\mu \mathrm{m}$ and $5-\mu \mathrm{m})$ with a diameter difference of $8 \mu \mathrm{m}$ can be separated, a finer separation resolution for particles with smaller size difference was also tested. From the particle diameter - electrical voltage map in Figure 5, for binary particle mixture $(8-\mu \mathrm{m}$ and $13-\mu \mathrm{m}$ in diameter), applying the electric voltage from $27 \mathrm{v}$ to $33 \mathrm{v}$, larger $(13-\mu \mathrm{m})$ particles are focused at the channel centerline, while the smaller $(8-\mu \mathrm{m})$ particles are still focused as two streaks close to the sidewalls, therefore, effective separation can be obtained, Figure S3. Regarding the limitations of the platform, the current design requires a top sheath flow to pinch all the particles down to the bottom region of microchannel, so that particles can experience the DEP force generated by the microelectrodes on the bottom surface. However, introduction of an additional sheath flow indeed complicates the operation of the system, dilutes the particle sample and consumes additional buffer. In the future work, we will try to optimize the design to eliminate the requirement of top sheath flow to further simplify the system.

Compared with previous works that the effective functioning of inertial microfluidic devices depends solely on flow rate $[20,59,61]$, the proposed DEP-inertial microfluidic platform not only keeps the functionality in particle separation by tuning flow rate but also adds one more flexible controlling parameter - electrical voltage to achieve particle separation. Furthermore, the proposed DEP-inertial microfluidic device can be tuned real-time to satisfy binary particle mixtures of varied sizes, without redesign of microchannel structure or dimension. We believed that this is a significant advantage of the proposed hybrid DEP-inertial microfluidic platform compared with the conventional inertial microfluidic devices.

\section{Conclusions}

In this work, we developed a novel DEP-inertial microfluidic device for particle separation. A sheath flow was used to successfully eliminate the top inertial focused streaks, so that the DEP force induced by the bottom patterned interdigitated microelectrodes can effectively exert on the microparticles and modified their focused positions within the channel cross-sections. The working mechanism of the proposed DEP-inertial microfluidic device was analyzed and its separation performance was evaluated by binary particle mixtures. In contrast to the traditional 
inertial microfluidic platforms, whose functionality highly depends on the flow speed, and they are only effective for a specific range target particle dimension, the superior advantage of the proposed DEP-inertial microfluidic devices is that the dimensions of target binary particle mixture can be tuned in real-time by simply adjusting the electric voltage, without the need of redesigning channel structure or dimension.

\section{Acknowledgements}

J. Zhang greatly acknowledges the support from the National Natural Science Foundation of China (Grant No.51705257) and the Natural Science Foundation of Jiangsu Province (Grant No. BK20170839). N.T. Nguyen and W.H. Li acknowledge the support from the Australian Research Council (ARC) Discovery Project (Grant No. DP180100055). S.H Tan acknowledges the support from the Australian Research Council (ARC) DECRA fellowship (Grant No. DE170100600).

\section{References}

[1] D. Pappas and K. Wang, "Cellular separations: a review of new challenges in analytical chemistry," Analytica Chimica Acta, vol. 601, pp. 26-35, 2007.

[2] Y. Gao, W. Li, and D. Pappas, "Recent advances in microfluidic cell separations," Analyst, vol. 138, pp. 4714-21, 2013.

[3] A. A. S. Bhagat, H. W. Hou, L. D. Li, C. T. Lim, and J. Han, "Pinched flow coupled shearmodulated inertial microfluidics for high-throughput rare blood cell separation," Lab on a Chip, vol. 11, pp. 1870-1878, 2011.

[4] D. Di Carlo, F. Jon, D. Irimia, R. G. Tompkins, and M. Toner, "Equilibrium separation and filtration of particles using differential inertial focusing," Analytical Chemistry, vol. 80, pp. 22042211, 2008.

[5] J. C.-T. Lin, D.-J. Lee, and C. Huang, "Membrane Fouling Mitigation: Membrane Cleaning," Separation Science \& Technology, vol. 45, pp. 858-872, 2010.

[6] J. Yao, H. Obara, A. Sapkota, and M. Takei, "Development of three-dimensional integrated microchannel-electrode system to understand the particles' movement with electrokinetics," Biomicrofluidics, vol. 10, p. 024105, 2016.

[7] W. Zhao, R. Cheng, J. R. Miller, and L. Mao, "Label-Free Microfluidic Manipulation of Particles and Cells in Magnetic Liquids," Advanced Functional Materials, vol. 26, pp. 3916-3932, 2016.

[8] Z. Wang and J. Zhe, "Recent advances in particle and droplet manipulation for lab-on-a-chip devices based on surface acoustic waves," Lab on a Chip, vol. 11, pp. 1280-1285, 2011.

[9] D. G. Grier, "A revolution in optical manipulation," Nature, vol. 424, pp. 810-816, 2003.

[10] L. R. Huang, E. C. Cox, R. H. Austin, and J. C. Sturm, "Continuous particle separation through deterministic lateral displacement," Science, vol. 304, pp. 987-990, 2004.

[11] M. Yamada, M. Nakashima, and M. Seki, "Pinched flow fractionation: continuous size separation of particles utilizing a laminar flow profile in a pinched microchannel," Analytical chemistry, vol. 76, pp. 5465-5471, 2004.

[12] D. Di Carlo, "Inertial microfluidics," Lab on a Chip, vol. 9, pp. 3038-3046, 2009.

[13] J. Zhang, S. Yan, D. Yuan, G. Alici, N.-T. Nguyen, M. Ebrahimi Warkiani, et al., "Fundamentals and applications of inertial microfluidics: a review," Lab on a Chip, vol. 16, pp. 10-34, 2016. 
[14] J. M. Martel and M. Toner, "Inertial Focusing in Microfluidics," Annual review of biomedical engineering, vol. 16, pp. 371-396, 2014.

[15] J. Zhou, P. V. Giridhar, S. Kasper, and I. Papautsky, "Modulation of aspect ratio for complete separation in an inertial microfluidic channel," Lab on a Chip, vol. 13, pp. 1919-1929, 2013.

[16] J. A. Kim, J. Lee, C. Wu, S. Nam, D. Di Carlo, and W. Lee, "Inertial focusing in non-rectangular cross-section microchannels and manipulation of accessible focusing positions," Lab on a Chip, vol. 16, pp. 992-1001, 2016.

[17] M. Rafeie, J. Zhang, M. Asadnia, W. Li, and M. E. Warkiani, "Multiplexing slanted spiral microchannels for ultra-fast blood plasma separation," Lab on a Chip, vol. 16, pp. 2791-802, 2016.

[18] J. Sun, M. Li, C. Liu, Y. Zhang, D. Liu, W. Liu, et al., "Double spiral microchannel for label-free tumor cell separation and enrichment," Lab on a chip, vol. 12, pp. 3952-3960, 2012.

[19] A. A. S. Bhagat, S. S. Kuntaegowdanahalli, and I. Papautsky, "Continuous particle separation in spiral microchannels using dean flows and differential migration," Lab Chip, vol. 8, pp. 19061914, 2008.

[20] J. Zhang, S. Yan, R. Sluyter, W. Li, G. Alici, and N.-T. Nguyen, "Inertial particle separation by differential equilibrium positions in a symmetrical serpentine micro-channel," Scientific reports, vol. 4, p. Art No. 4527, 2014.

[21] D. Di Carlo, D. Irimia, R. G. Tompkins, and M. Toner, "Continuous inertial focusing, ordering, and separation of particles in microchannels," Proceedings of the National Academy of Sciences, vol. 104, pp. 18892-18897, 2007.

[22] M. G. Lee, J. H. Shin, C. Y. Bae, S. Choi, and J.-K. Park, "Label-free cancer cell separation from human whole blood using inertial microfluidics at low shear stress," Analytical chemistry, vol. 85, pp. 6213-6218, 2013.

[23] Z. Wu, Y. Chen, M. Wang, and A. J. Chung, "Continuous inertial microparticle and blood cell separation in straight channels with local microstructures," Lab on a Chip, vol. 16, pp. 532-542, 2016.

[24] E. Sollier, D. E. Go, J. Che, D. R. Gossett, S. O'Byrne, W. M. Weaver, et al., "Size-selective collection of circulating tumor cells using Vortex technology," Lab on a Chip, vol. 14, pp. 63-77, 2014

[25] X. Wang and I. Papautsky, "Size-based microfluidic multimodal microparticle sorter," Lab on a Chip, vol. 15, pp. 1350-1359, 2015.

[26] D. Stoecklein, C.-Y. Wu, K. Owsley, Y. Xie, D. Di Carlo, and B. Ganapathysubramanian, "Micropillar sequence designs for fundamental inertial flow transformations," Lab on a Chip, vol. 14, pp. 4197-4204, 2014.

[27] H. Amini, E. Sollier, M. Masaeli, Y. Xie, B. Ganapathysubramanian, H. A. Stone, et al., "Engineering fluid flow using sequenced microstructures," Nature communications, vol. 4, p. Art No. 1826, 2013.

[28] M. G. Lee, S. Choi, H. J. Kim, H. K. Lim, J. H. Kim, N. Huh, et al., "Inertial blood plasma separation in a contraction-expansion array microchannel," Applied Physics Letters, vol. 98, p. $253702,2011$.

[29] S. S. Kuntaegowdanahalli, A. A. S. Bhagat, G. Kumar, and I. Papautsky, "Inertial microfluidics for continuous particle separation in spiral microchannels," Lab Chip, vol. 9, pp. 2973-2980, 2009.

[30] L. Wu, G. Guan, H. W. Hou, A. A. S. Bhagat, and J. Han, "Separation of Leukocytes from Blood Using Spiral Channel with Trapezoid Cross-Section," Analytical Chemistry, vol. 84, pp. 93249331, 2012.

[31] E. Sollier, H. Amini, D. Go, P. Sandoz, K. Owsley, and D. Di Carlo, "Inertial microfluidic programming of microparticle-laden flows for solution transfer around cells and particles," Microfluidics and Nanofluidics, vol. 19, pp. 53-65, 2015. 
[32] D. R. Gossett, H. T. K. Tse, J. S. Dudani, K. Goda, T. A. Woods, S. W. Graves, et al., "Inertial manipulation and transfer of microparticles across laminar fluid streams," Small, vol. 8, pp. 27572764, 2012.

[33] J. M. Martel, K. C. Smith, M. Dlamini, K. Pletcher, J. Yang, M. Karabacak, et al., "Continuous Flow Microfluidic Bioparticle Concentrator," Scientific Reports, vol. 5, p. 11300, 2015.

[34] H. W. Hou, M. E. Warkiani, B. L. Khoo, Z. R. Li, R. A. Soo, D. S.-W. Tan, et al., "Isolation and retrieval of circulating tumor cells using centrifugal forces," Scientific reports, vol. 3, p. Art No. 1259, 2013.

[35] M. E. Warkiani, G. Guan, K. B. Luan, W. C. Lee, A. A. S. Bhagat, P. K. Chaudhuri, et al., "Slanted spiral microfluidics for the ultra-fast, label-free isolation of circulating tumor cells," Lab on a Chip, vol. 14, pp. 128-137, 2014.

[36] E. Ozkumur, A. M. Shah, J. C. Ciciliano, B. L. Emmink, D. T. Miyamoto, E. Brachtel, et al., "Inertial focusing for tumor antigen-dependent and-independent sorting of rare circulating tumor cells," Science translational medicine, vol. 5, p. 179ra47, 2013.

[37] M. E. Warkiani, A. K. P. Tay, B. L. Khoo, X. Xiaofeng, J. Han, and C. T. Lim, "Malaria detection using inertial microfluidics," Lab on a Chip, vol. 15, pp. 1101-1109, 2015.

[38] J. K. Nunes, C. Y. Wu, H. Amini, K. Owsley, D. Di Carlo, and H. A. Stone, "Fabricating Shaped Microfibers with Inertial Microfluidics," Advanced Materials, vol. 26, pp. 3712-3717, 2014.

[39] W. C. Lee, A. A. S. Bhagat, S. Huang, K. J. Van Vliet, J. Han, and C. T. Lim, "High-throughput cell cycle synchronization using inertial forces in spiral microchannels," Lab on a Chip, vol. 11, pp. 1359-1367, 2011.

[40] E. W. Kemna, R. M. Schoeman, F. Wolbers, I. Vermes, D. A. Weitz, and A. van den Berg, "High-yield cell ordering and deterministic cell-in-droplet encapsulation using Dean flow in a curved microchannel," Lab on a Chip, vol. 12, pp. 2881-2887, 2012.

[41] D. R. Gossett, T. Henry, S. A. Lee, Y. Ying, A. G. Lindgren, O. O. Yang, et al., "Hydrodynamic stretching of single cells for large population mechanical phenotyping," Proceedings of the National Academy of Sciences, vol. 109, pp. 7630-7635, 2012.

[42] S. Yan, J. Zhang, D. Yuan, and W. Li, "Hybrid microfluidics combined with active and passive approaches for continuous cell separation," Electrophoresis, vol. 38, pp. 238-249, 2017.

[43] J. P. Beech, P. Jönsson, and J. O. Tegenfeldt, "Tipping the balance of deterministic lateral displacement devices using dielectrophoresis," Lab on A Chip, vol. 9, pp. 2698-2706, 2009.

[44] S. Yan, J. Zhang, G. Alici, H. Du, Y. Zhu, and W. Li, "Isolating plasma from blood using a dielectrophoresis-active hydrophoretic device," Lab on a Chip, vol. 14, pp. 2993-3003, 2014.

[45] P. R. C. Gascoyne, "Dielectrophoretic-Field Flow Fractionation Analysis of Dielectric, Density, and Deformability Characteristics of Cells and Particles," Analytical Chemistry, vol. 81, pp. 8878-8885, 2009/11/01 2009.

[46] M. Mizuno, M. Yamada, R. Mitamura, K. Ike, K. Toyama, and M. Seki, "Magnetophoresisintegrated hydrodynamic filtration system for size- and surface marker-based two-dimensional cell sorting," Analytical Chemistry, vol. 85, pp. 7666-73, 2013.

[47] F. Del Giudice, H. Madadi, M. M. Villone, G. D'Avino, A. M. Cusano, R. Vecchione, et al., "Magnetophoresis 'meets' viscoelasticity: deterministic separation of magnetic particles in a modular microfluidic device," Lab on a Chip, vol. 15, pp. 1912-1922, 2015.

[48] J. Zhang, S. Yan, D. Yuan, Q. Zhao, S. H. Tan, N. T. Nguyen, et al., "A novel viscoelastic-based ferrofluid for continuous sheathless microfluidic separation of nonmagnetic microparticles," Lab on A Chip, vol. 16, pp. 3947-3956, 2016.

[49] D. J. Collins, T. Alan, and A. Neild, "Particle separation using virtual deterministic lateral displacement (vDLD)," Lab on a Chip, vol. 14, pp. 1595-1603, 2014.

[50] F. Fachin, P. Spuhler, J. M. Martel-Foley, J. F. Edd, T. A. Barber, J. Walsh, et al., "Monolithic Chip for High-throughput Blood Cell Depletion to Sort Rare Circulating Tumor Cells," Scientific Reports, vol. 7, p. 10936, 2017. 
[51] Y. Zhou, L. Song, L. Yu, and X. Xuan, "Inertially focused diamagnetic particle separation in ferrofluids," Microfluidics and Nanofluidics, vol. 21, p. 14, 2017.

[52] H. S. Moon, K. Kwon, S. I. Kim, H. Han, J. Sohn, S. Lee, et al., "Continuous separation of breast cancer cells from blood samples using multi-orifice flow fractionation (MOFF) and dielectrophoresis (DEP)," Lab on a Chip, vol. 11, pp. 1118-1125, 2011.

[53] N. M. Karabacak, P. S. Spuhler, F. Fachin, E. J. Lim, V. Pai, E. Ozkumur, et al., "Microfluidic, marker-free isolation of circulating tumor cells from blood samples," Nature protocols, vol. 9, pp. 694-710, 2014.

[54] J. Zhang, S. Yan, G. Alici, N.-T. Nguyen, D. Di Carlo, and W. Li, "Real-time control of inertial focusing in microfluidics using dielectrophoresis (DEP)," RSC Advances, vol. 4, pp. 6207662085, 2014.

[55] J. Zhou and I. Papautsky, "Fundamentals of inertial focusing in microchannels," Lab on a Chip, vol. 13, pp. 1121-1132, 2013.

[56] Y. Xia and G. M. Whitesides, "Soft lithography," Annual review of materials science, vol. 28, pp. 153-184, 1998.

[57] M. Li, S. Li, J. Wu, W. Wen, W. Li, and G. Alici, "A simple and cost-effective method for fabrication of integrated electronic-microfluidic devices using a laser-patterned PDMS layer," Microfluidics and nanofluidics, vol. 12, pp. 751-760 2012.

[58] S. H. Baek, W.-J. Chang, J.-Y. Baek, D. S. Yoon, R. Bashir, and S. W. Lee, "Dielectrophoretic technique for measurement of chemical and biological interactions," Analytical chemistry, vol. 81, pp. 7737-7742, 2009.

[59] G. Guan, L. Wu, A. A. Bhagat, Z. Li, P. C. Chen, S. Chao, et al., "Spiral microchannel with rectangular and trapezoidal cross-sections for size based particle separation," Scientific reports, vol. 3, p. 1475, 2013.

[60] M. G. Lee, S. Choi, and J. K. Park, "Inertial separation in a contraction-expansion array microchannel," Journal of Chromatography A, vol. 1218, pp. 4138-4143, 2011.

[61] R. Khojah, R. Stoutamore, and D. Di Carlo, "Size-tunable microvortex capture of rare cells," Lab on A Chip, vol. 17, pp. 2542-2549, 2017. 


\section{Author Biography}

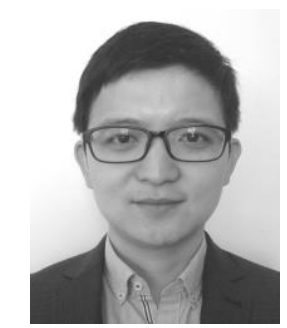

Jun Zhang received his bachelor degree in Engineering from the Nanjing University of Science and Technology (NJUST), Nanjing, China in 2009 with an Outstanding Graduate Award and received a Ph.D. degree in Mechanical Engineering from the University of Wollongong, Australia in 2015. After that, he conducted his postdoctoral research as a research scientist and an associate research fellow at Woolcock Institute of Medical Research and the University of Wollongong, respectively. He is currently an Associate Professor at Nanjing University of Science and Technology (NJUST), China. He has published more than 30 journal and conference articles.

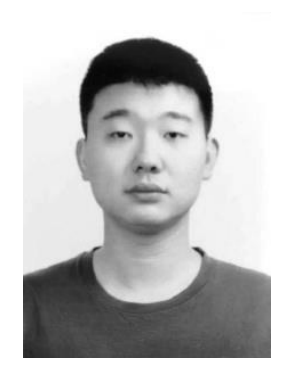

Qianbin Zhao received his bachelor's degree in engineering from the Northeastern University (NEU), Shenyang, China, in 2015. He recently holds a position as a Ph.D. candidate in the School of Mechanical, Materials, Mechatronic and Biomedical Engineering at the University of Wollongong. His current research focuses on (i) inertial microfluidics for microparticle manipulation through geometry-induced secondary flow and (ii) viscoelastic microfluidics applications for bioassays and biosample processing. 


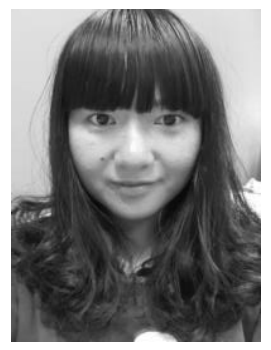

Dan Yuan was born in Shandong province, China, in 1990. She received her Bachelor degree in Engineering and Master degrees in Engineering from Hefei University of Technology in Hefei, China, in 2011 and 2014, and majored in Precision Measurement Technology and Instruments.

Since 2014, she has been a PhD candidate in the School of Mechanical, Materials, Mechatronic and Biomedical Engineering at University of Wollongong. She is the author of more than 18 papers. Her research interests are particle and cell manipulation in viscoelastic fluid.

Sheng Yan received his Bachelor degree in Engineering with an Outstanding Graduate Award from China University of Mining and Technology in 2012 and a doctoral degree from the University of Wollongong in 2016. He currently works as an associate research fellow in the School of Mechanical, Materials and Mechatronic Engineering at the University of Wollongong. His main research focus is to develop hybrid techniques to tune hydrophoresis for cell focusing and separation. His research interests include dielectrophoresis, hydrophoresis, magnetophoresis, neurosciences, computational fluid dynamics (CFD).

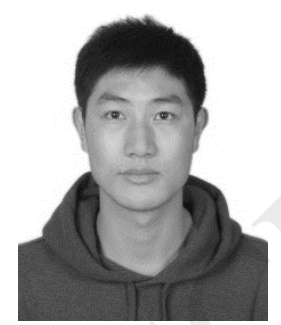

Dr. Shiyang Tang currently is a Vice-Chancellor's Postdoctoral Fellow at the University of Wollongong, Australia. He received his $\mathrm{PhD}$ in Microelectromechanical Systems (MEMS) from RMIT University, Australia in 2015. He joined Pennsylvania State University, USA, as a postdoctoral research fellow from Aug 2015 to Jul 2016, working on surface acoustic wave enabled microfluidic platforms. Later he joined the University of California, San Francisco (UCSF), USA, as a postdoctoral scholar from Aug 2016 to Feb 2017, working on droplet microfluidics for point-of-care diagnosis. His research interests include developing microfluidic platforms for

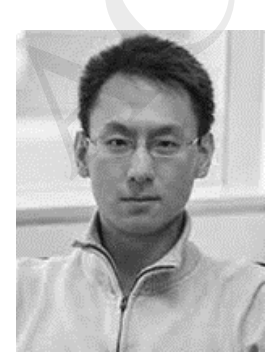
biomedical studies and liquid metal enabled micro-/nanoscale platforms. He is the named author of 39 papers in different peer-reviewed journals, including PNAS, Advanced Materials, Advanced Functional Materials, Small, Analytical Chemistry, and Lab on a Chip. The outcomes of his works have been highlighted in many world-known science magazines, such as Nature, RSC Chemistry World, New Scientists, Science Daily, Physics Org, Nanowerk, and Material Views. 


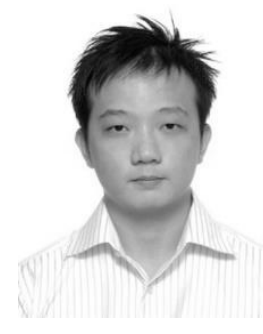

Say Hwa Tan is an ARC DECRA fellow with Queensland Micro-and Nanotechnology Centre, Griffith University, Australia. He received his BEng, MEng and PhD degrees from the Nanyang Technological University, Singapore, and the Georg-August-Universität Göttingen/Max Planck Institute for dynamics and self-organization (MPI-DS), Germany, in 2008, 2010 and 2014 respectively. In 2016, he was highlighted as one of the 18 emerging investigators in the journal of Lab on a Chip. Dr Tan has published more than 30 research works in microfluidics. His research has established and pioneered different approaches to manipulate droplets and bubbles using thermal, magnetic, acoustic, pneumatic and electric energy.

Jinhong Guo received the Bachelor's degree in electronic engineering from the University of Electronic Science and

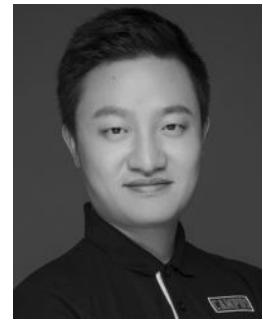
Technology of China, Chengdu, China, in 2010 and the Ph.D. degree in biomedical engineering from the Nanyang Technological University, Singapore, in 2014. He is currently a Full Professor with the School of Communication and Information Engineering, University of Electronic Science and Technology of China, Chengdu, China. After his doctoral studies, during 2014-2015, he was a Postdoctoral Fellow in the Pillar of Engineering Design with MIT-SUTD, Singapore. From January 2016 to July 2016, he was a Visiting Professor with the School of Mechanical Engineering, University of Michigan, Ann Arbor, MI, USA. His current research interests include electrochemical sensor and lab-on-a-chip devices for point of care test toward clinical use. He has authored or coauthored 70 publication in top journal, such as IEEE TII, Analytical Chemistry, Biosensor, and Bioelectronics, etc.

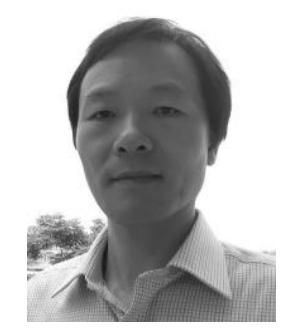

Huanming Xia is currently in the School of Mechanical Engineering, Nanjing University of Science and Technology. He received his B.Eng. and M.Eng. degrees in thermal engineering from Nanjing University of Aeronautics \& Astronautics, China, 1998, 2001, and a Ph.D. degree from National University of Singapore, Singapore, 2010. From 2007 to 2014, he worked in Singapore Institute of Manufacturing Technology (SIMTech) as a Research Scientist. His research interests include fluid dynamics, heat and mass transfer, microfluidics, microreactor technology and micro-fabrication techniques.

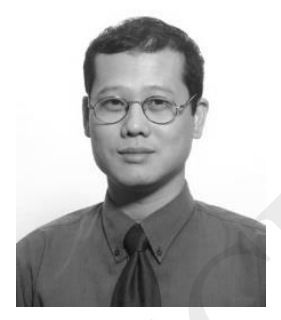

Nam-Trung Nguyen received his Dip-Ing, Dr. Ing and Dr. Ing Habil degrees from Chemnitz University of Technology, Germany, in 1993, 1997 and 2004, respectively. The habilitation degree (Dr. Ing Habil) is the qualification for a full professorship in Germany. In 1998, he was a postdoctoral research engineer in the Berkeley Sensor and Actuator Center (University of California at Berkeley, USA). Prof Nguyen is the First Runner Up of Inaugural ProSPER.Net-Scopus Young Scientist Awards in Sustainable Development in 2009 and the Runner Up of ASAIHL-Scopus Young Scientist Awards in 2008. He is a Fellow of ASME and a Member of IEEE. Nguyen's research is focused on microfluidics, nanofluidics, micro/nanomachining technologies, micro/nanoscale science, and instrumentation for biomedical applications. He published over 300 journal papers and filed 8 patents, of which 3 were granted. Among the books he has written, the first and second editions of the bestseller "Fundamentals and Applications of Microfluidics" were published in 2002 and 2006, respectively. The second edition of the bestselling book "Micromixer" was acquired and published by Elsevier in 2011. 


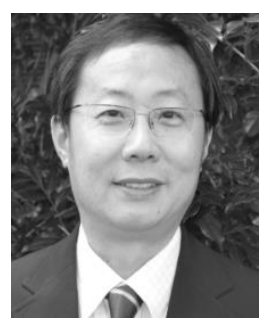

Weihua Li, Ph.D., is a Senior Professor and Director of the Advanced Manufacturing Research Strength at the University of Wollongong. He completed his BEng (1992), MEng (1995) at University of Science and Technology of China, and Ph.D. (2001) at Nanyang Technological University (NTU). He was with the School of Mechanical and Aerospace Engineering of NTU as a Research Associate/Fellow from 2000 to 2003, before he joined the School of Mechanical, Materials and Mechatronic Engineering as a Lecturer. His research focuses on smart materials and their applications, microfluidics, rheology, and intelligent mechatronics. He is serving as editor or editorial board member for several international journals, including IEEE/ASME Transactions on Mechatronics, Smart Materials and Structures, Scientific Reports, etc. He has published more than 320 journal and conference papers. He is a recipient of Fellow of the Engineers Australia, the Fellow of Institute of Physics (UK), Australian Endeavour Research Fellowship, JSPS Invitation Fellowship, Vice-chancellor's Award for Interdisciplinary Research Excellence, and numerous Best Paper Awards. 


\section{Figure captions}

Figure 1 Particle separation in the DEP-inertial microfluidic platform. (a) 3D illustration of the DEP-inertial microfluidic device for particle separation. (b) The cross-sectional view at the end of the microchannel. The small arrows indicate the vectors of the secondary flow within the cross section. (c) Side view illustrates particle trajectory along the height direction. (d) The photograph of the fabricated DEP-inertial microfluidic device.

Figure 2 Top sheath flow pushes all the particles into the bottom of the microchannel, and eliminates the top inertial focusing equilibrium position. (i-iv) Without sheath flow, particles are focused as two symmetrical streaks at both top and bottom of the microchannel; (v-viii) When sheath flow is applied with a flow rate ratio (sheath flow: sample flow) as $2: 1$, the top inertial focused streak vanishes. (ix) The fluorescence intensity profile along the height direction at the end of serpentine microchannel indicates that only one focused streak left after implementation of the top sheath flow. The total flow rate is $300 \mu 1 / \mathrm{min}$.

Figure 3 (a) DEP-inertial focusing of $8-\mu \mathrm{m}$ particles with an increasing electric voltage and (b) the corresponding fluorescence profiles clearly indicate the distribution of microparticles along the channel width. The flow rate ratio $=2: 1$, and the total flow rate $=300 \mu 1 / \mathrm{min}$.

Figure 4 (a) Transformation of DEP-inertial focusing patterns at an increasing electrical voltage and (b) the corresponding speculated dynamic equilibrium positions of particles within the microchannel cross-sections. (c) Force analysis of DEP force $F_{D E P}$ and inertial lift force $F_{L}$ along the vertical direction $(z)$ for the different inertial focusing patterns. The red arrows indicate the

direction of net force of $F_{D E P}$ and $F_{L}$. For a stable position, the net force should direct toward it along both sides. (I) At relative low electric voltages, particles are focused at location A along the vertical direction $(z)$. Point $C$ is an unstable position where the net force on both sides directs outwards and unstable along both positive and negative $z$ direction, thus particles can easily escape towards both sides by a weak disturbance. No particles are within the top half of 
microchannel at the inlet and thus no particles are focused at point D, although point D is a stable position. This stage includes the inertial focusing patterns (i-iii) in Figure 4(a); (II) Increasing electrical voltage, point A moves upwards and point $\mathrm{C}$ moves downwards, and eventually they contact and merge as a point B. Point B is a semi-stable (saddle) position, where the net force on both sides is directing upward. Due to the fact that point $\mathrm{B}$ is a semi-stable position, a portion of particles on point $\mathrm{B}$ may shift upward to point $\mathrm{D}$ and the remaining particles are staying at point B. Therefore, from top-down view, there are three focusing streaks, corresponding to focusing pattern (iv) in Figure 4(a); (iii) When the applied voltage exceeds a threshold, all the particles will move to point D, which corresponds to focusing pattern (v) in Figure 4(a).

Figure 5 The fluorescent images of $13-\mu \mathrm{m}$ polystyrene particle focusing streaks under different electric frequencies. $\mathrm{V}_{\mathrm{pp}}=30 \mathrm{v}$; (b) The theoretical Clausius-Mossotti (CM) factor under different electric frequencies.

Figure 6 The particle diameter - electrical voltage map illustrates the working regions of different particle focusing patterns. In region I, particles are focused along two sidewalls due to the weak DEP force; In region II, sufficient DEP force levitates particles to the vertical positions where particles can be pinched as a single focused streak at the channel center; Region I and region II correspond to the stage I in dynamic analysis. In region III, DEP force is too strong and the resultant of DEP force and inertial lift force cannot create stable positions within the bottom area of the channel, thus a large portion of and even all particles escape and migrate to the top stable positions as two sided streaks. Region III includes the stages II and III in the dynamic analysis. The critical electrical voltage $V_{C l}$ is the minimum voltage that the centrally focused streak is formed. The critical electrical voltage $V_{C 2}$ is the minimum voltage that a portion of particles move toward the top stable positions and two-sided focused streaks appear.

Figure 7 (a) Focusing positions and focusing widths of 5- $\mu \mathrm{m}$ and $13-\mu \mathrm{m}$ particles under different electrical voltages. (b) and (c) Binary particles $(13-\mu \mathrm{m}$ and $5-\mu \mathrm{m}$ in diameter) trajectories at $\mathrm{V}_{\mathrm{pp}}=0 \mathrm{v}$, and $\mathrm{V}_{\mathrm{pp}}=27 \mathrm{v}$, respectively. (i) The fluorescence trajectory at the last period, (ii) the 
fluorescence trajectory at the end of the serpentine microchannel, and (iii) the corresponding fluorescence profiles. Green fluorescent lines represent trajectories of $5-\mu \mathrm{m}$ particles and red fluorescent lines indicate trajectories of $13-\mu \mathrm{m}$ particles.

Figure 8 Separation of polystyrene beads ( $5 \mu \mathrm{m}$ and $13 \mu \mathrm{m}$ in diameter) in the DEP-inertial microfluidic device. (a) Fluorescent images of particle collection from inlet and two outlets under a hemocytometry. (b) Separation purity of binary particle mixture before and after separation. (c) Separation efficiency of the binary particle mixture of $5-\mu \mathrm{m}$ and $13-\mu \mathrm{m}$ particles. 
Fig 1

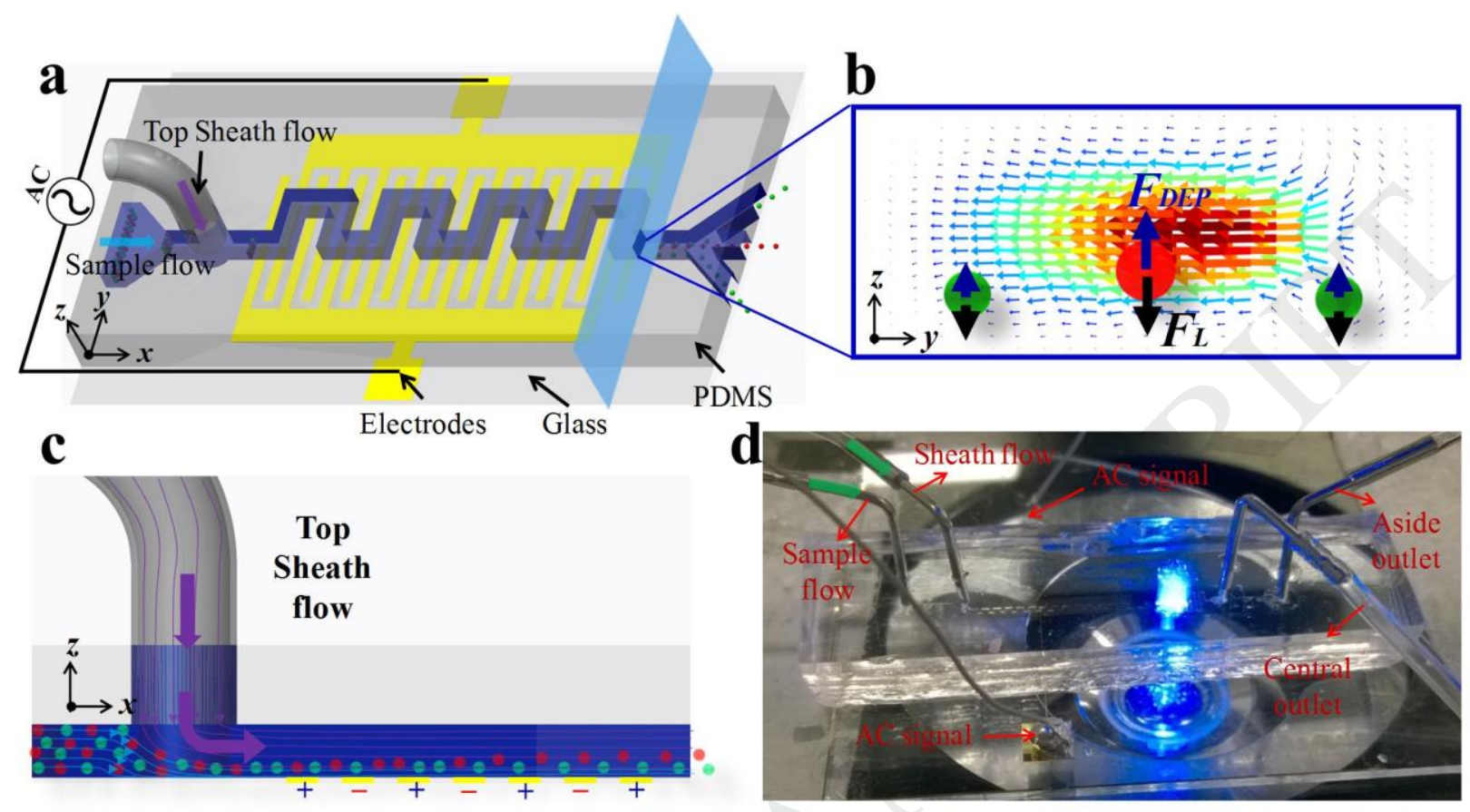


Fig 2
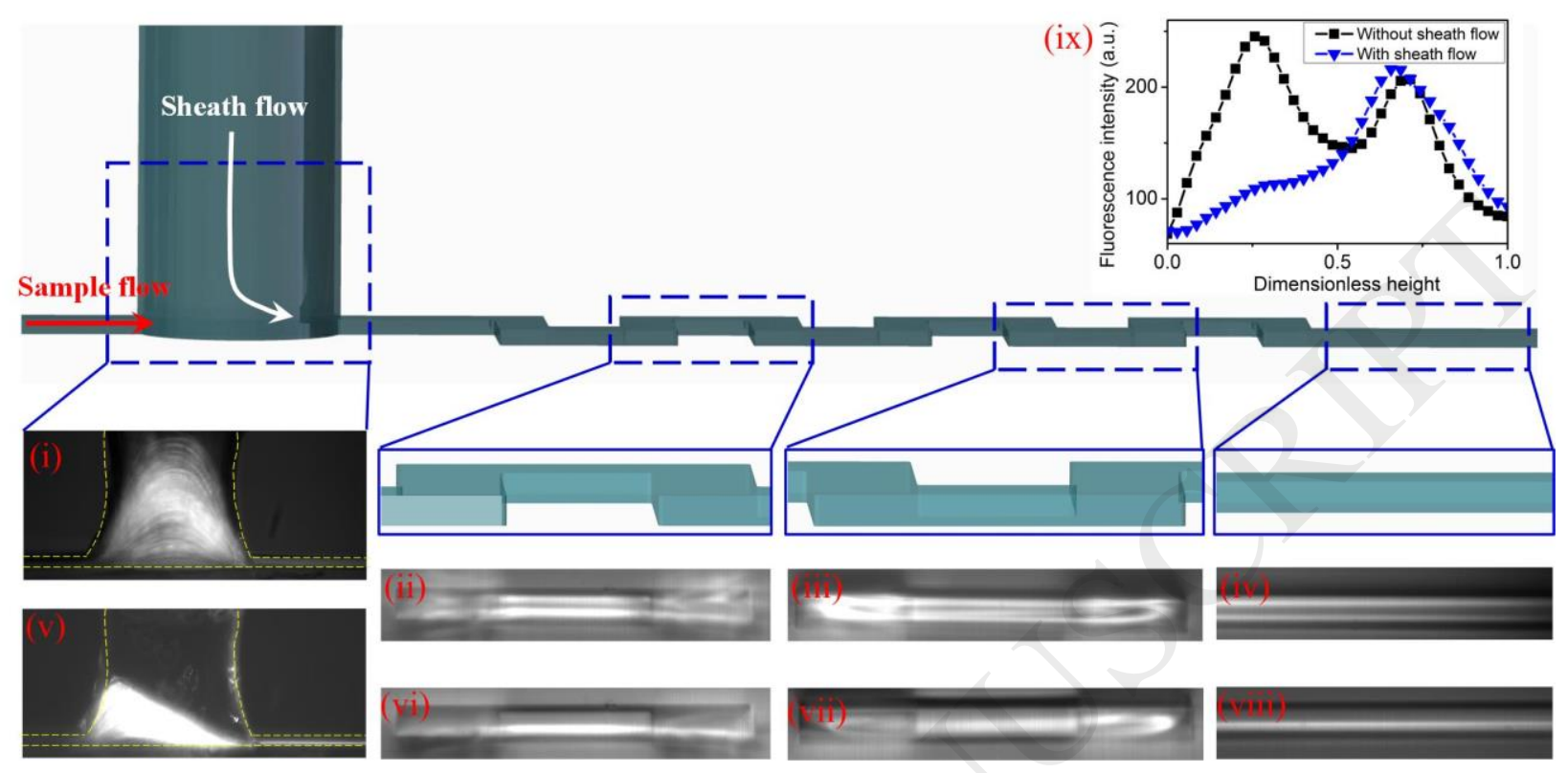
Fig 3

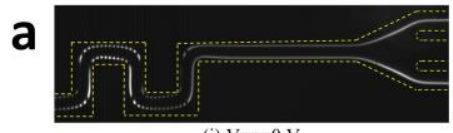

(i) $\mathrm{Vpp}=0 \mathrm{~V}$

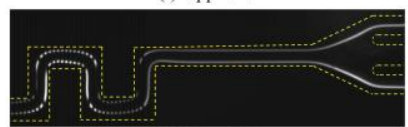

(ii) $\mathrm{Vpp}=9 \mathrm{~V}$

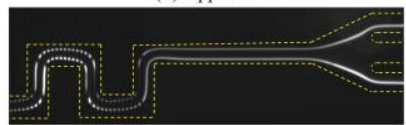

(iii) $\mathrm{Vpp}=21 \mathrm{~V}$

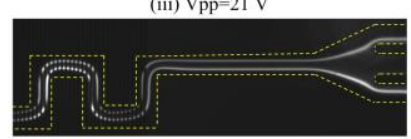

(iv) $\mathrm{Vpp}=27 \mathrm{~V}$

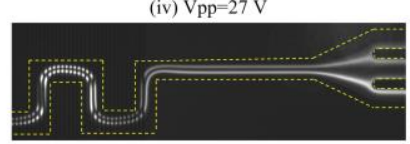

(v) $V p p=33 \mathrm{~V}$

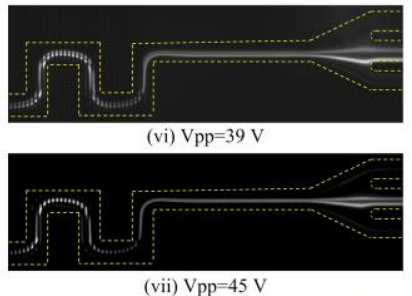

(vii) $\mathrm{Vpp}=45 \mathrm{~V}$

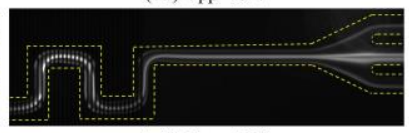

(viii) $\mathrm{Vpp}=48 \mathrm{~V}$

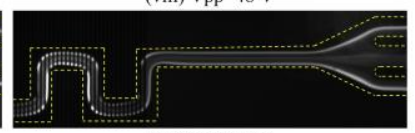

(ix) $\mathrm{Vpp}=54 \mathrm{~V}$

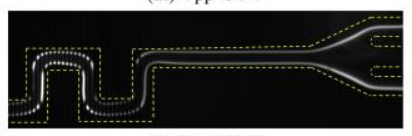

(x) $\mathrm{Vpp}=60 \mathrm{~V}$

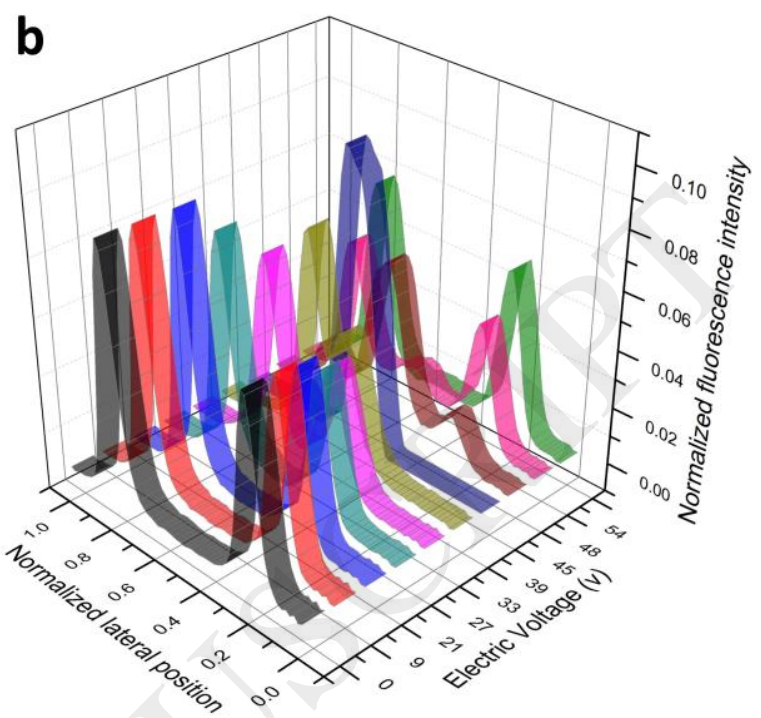


Fig 4

(a)
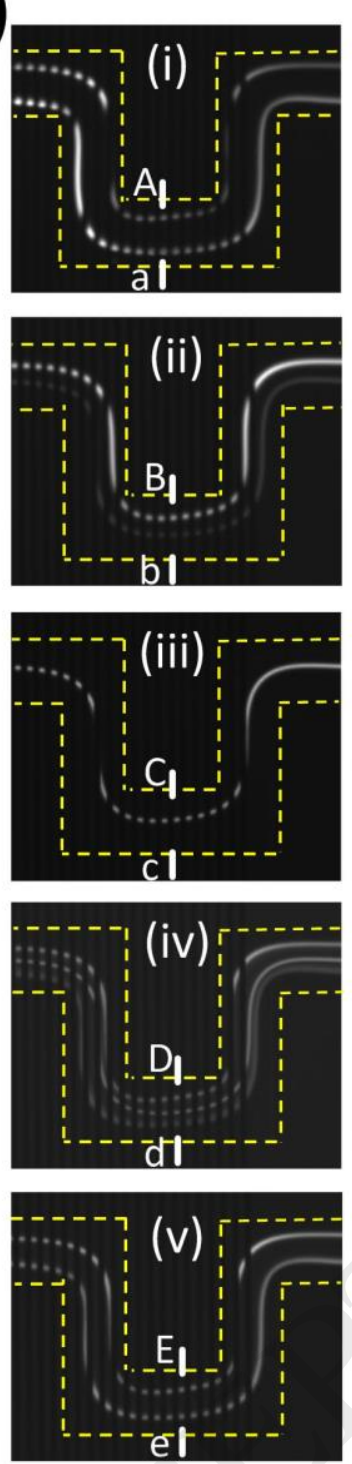

(b)
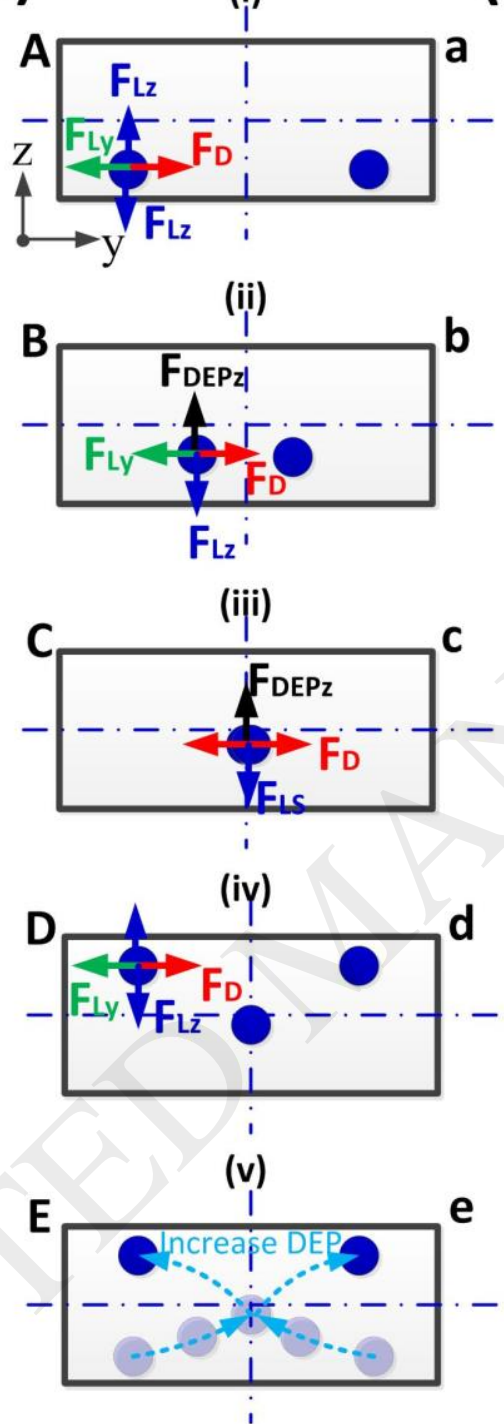

(c)
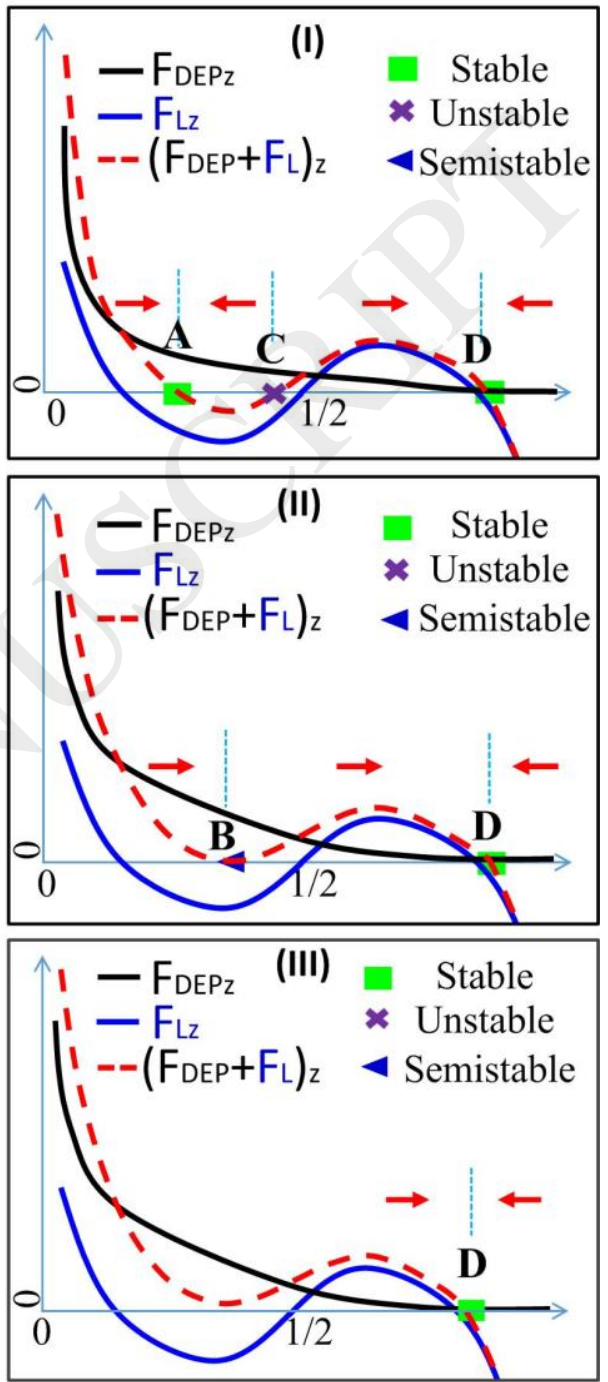

Normalized height $(z / H)$ 
Fig 5

a

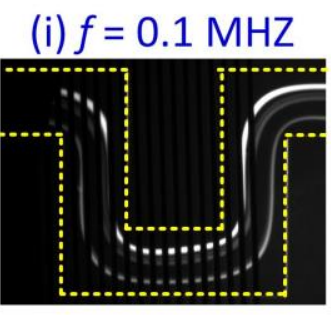

(iv) $f=0.5 \mathrm{MHZ}$

(vii) $f=10 \mathrm{MHZ}$
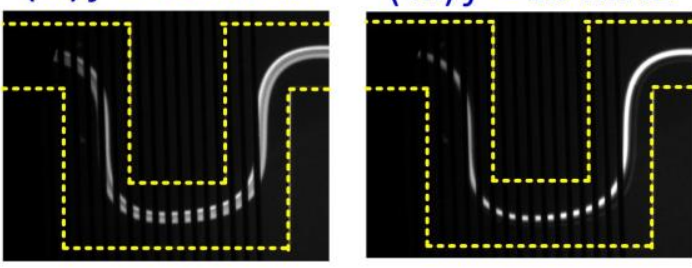

(ii) $f=0.2 \mathrm{MHZ}$

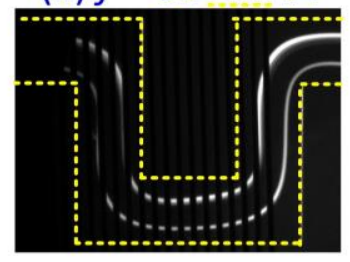

(v) $f=1 \mathrm{MHZ}$

(viii) $f=20 \mathrm{MHZ}$
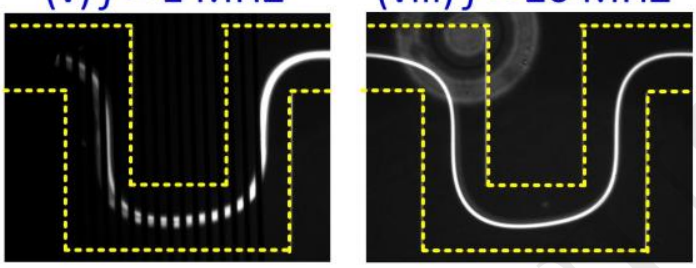

(iii) $f=0.3 \mathrm{MHZ}$

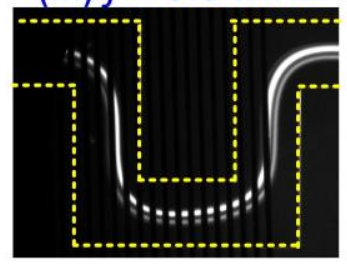

(vi) $f=5 \mathrm{MHZ}$

(ix) $f=30 \mathrm{MHZ}$
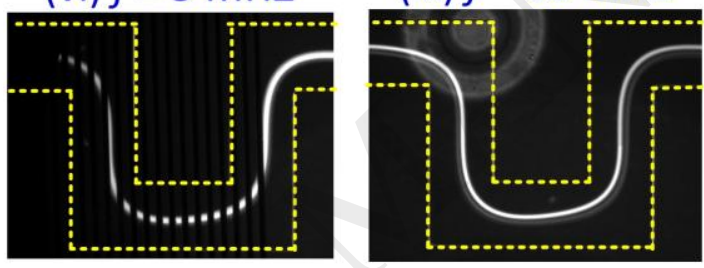

b

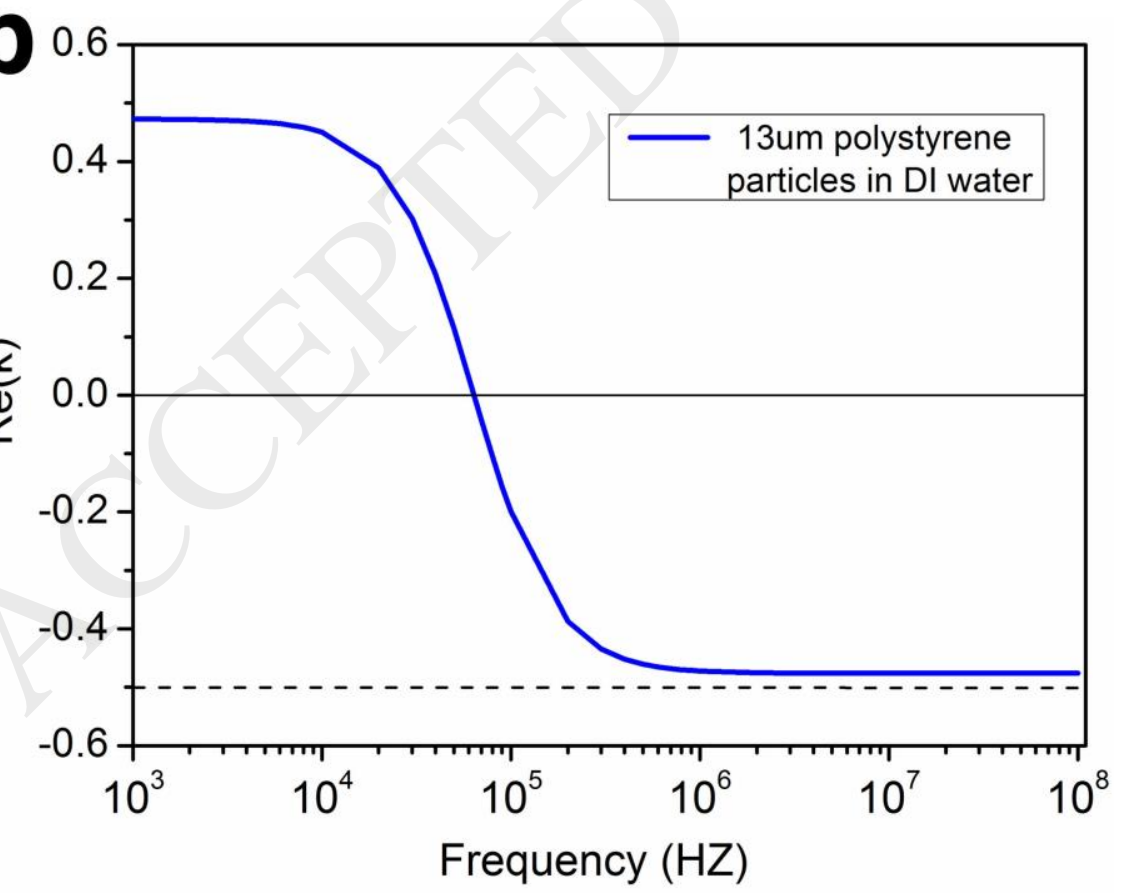


Fig 6

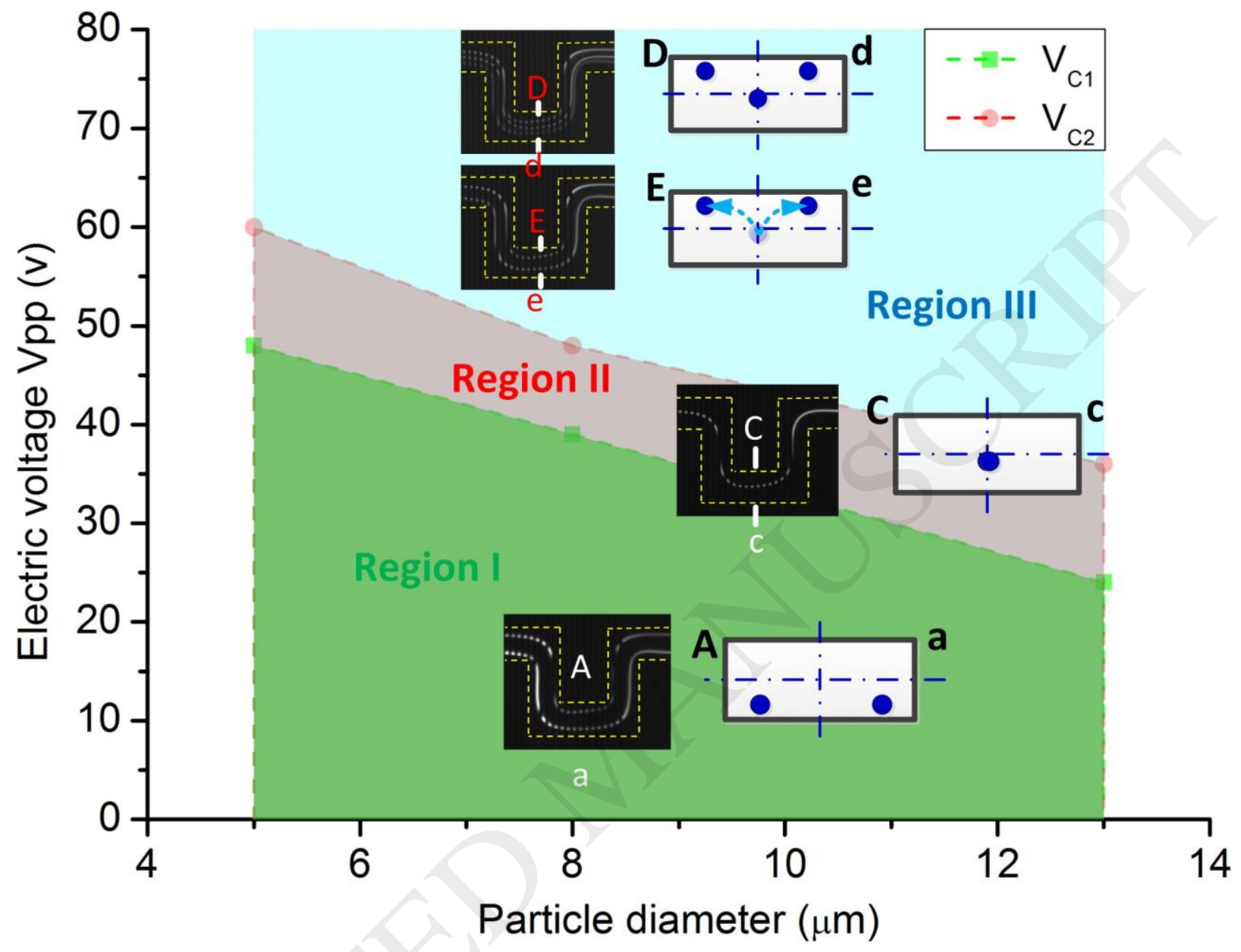


Fig 7
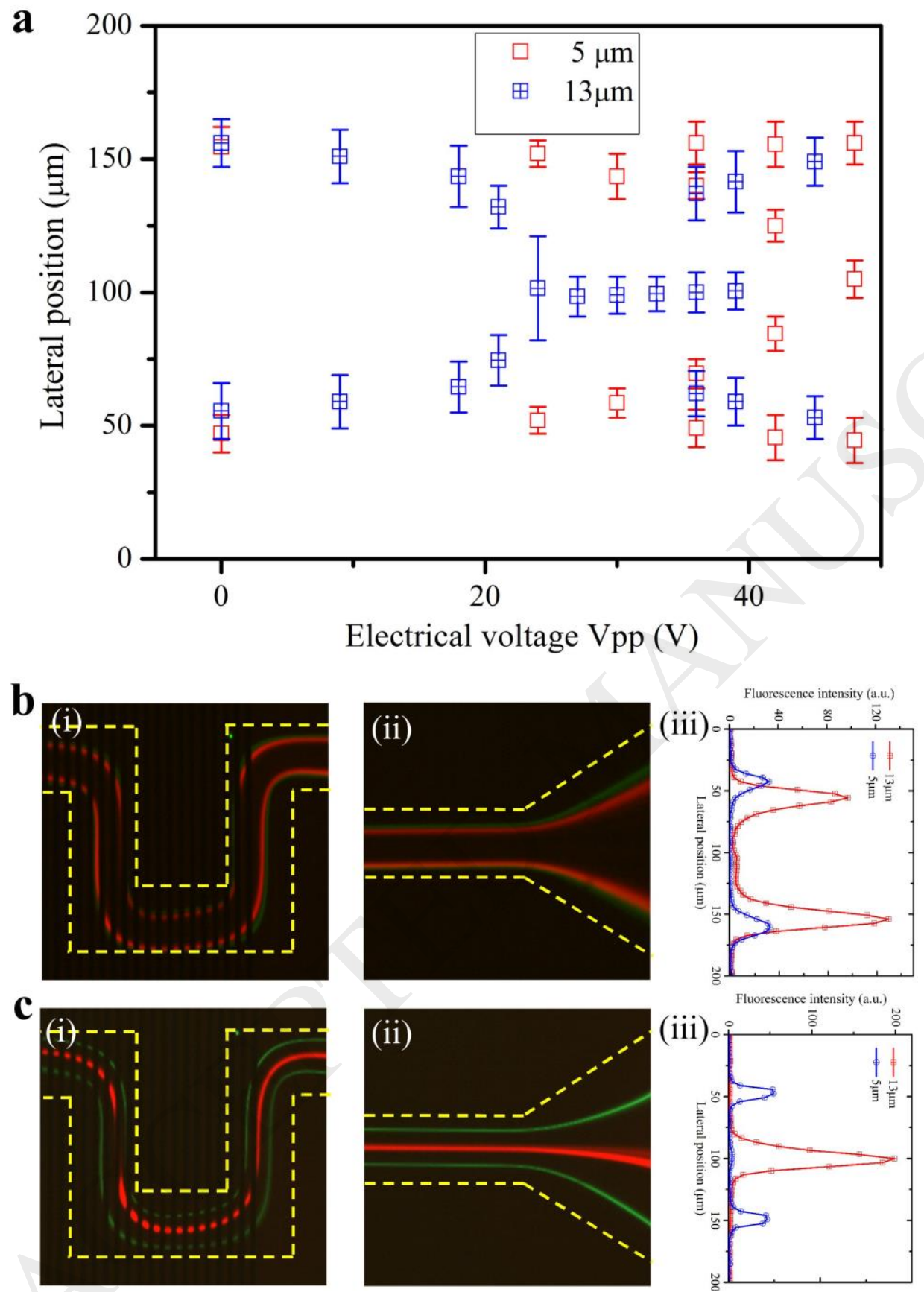
Fig 8

d
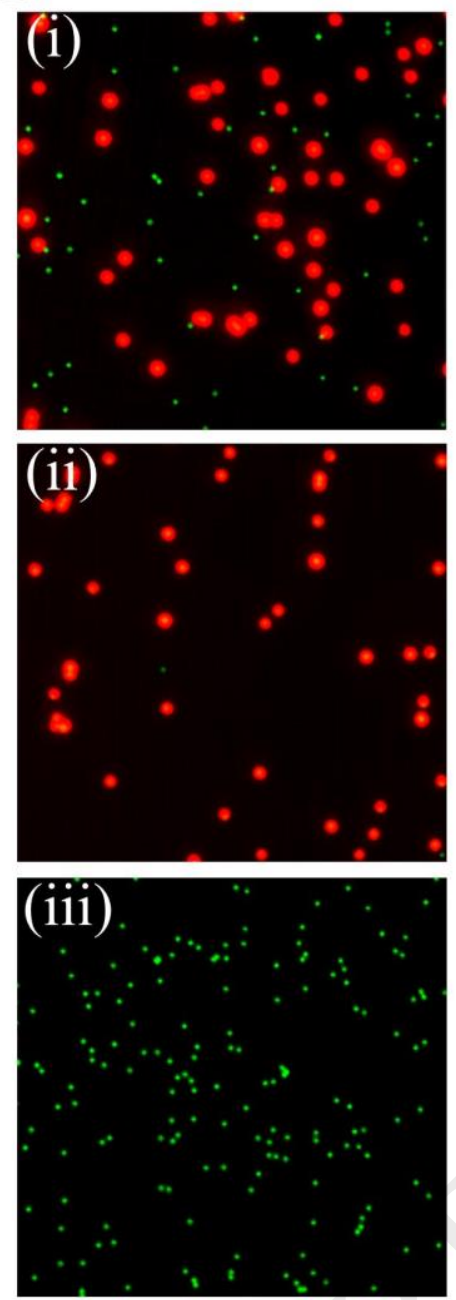

b
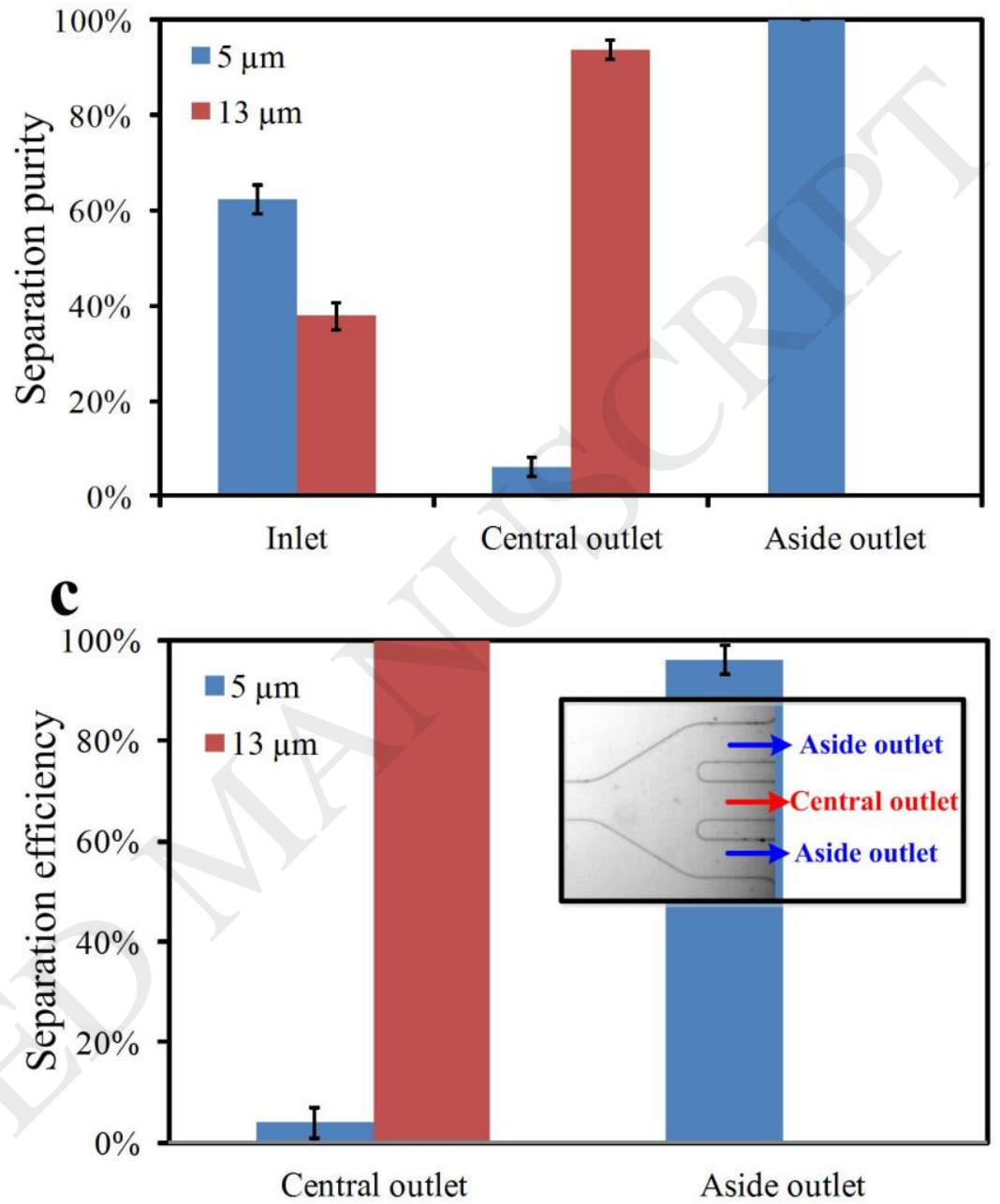


\section{Accepted Manuscript}

Title: Tunable particle separation in a hybrid dielectrophoresis (DEP)- inertial microfluidic device

Authors: Jun Zhang, Dan Yuan, Qianbin Zhao, Sheng Yan, Shi-Yang Tang, Say Hwa Tan, Jinhong Guo, Huanming Xia, Nam-Trung Nguyen, Weihua Li

PII: S0925-4005(18)30701-9

DOI: https://doi.org/10.1016/j.snb.2018.04.020

Reference: SNB 24495

To appear in: Sensors and Actuators B

Received date: $\quad$ 20-11-2017

Revised date: $\quad 1-3-2018$

Accepted date: $\quad$ 2-4-2018

Please cite this article as: Jun Zhang, Dan Yuan, Qianbin Zhao, Sheng Yan, Shi-Yang Tang, Say Hwa Tan, Jinhong Guo, Huanming Xia, Nam-Trung Nguyen, Weihua Li, Tunable particle separation in a hybrid dielectrophoresis (DEP)- inertial microfluidic device, Sensors and Actuators B: Chemical https://doi.org/10.1016/j.snb.2018.04.020

This is a PDF file of an unedited manuscript that has been accepted for publication. As a service to our customers we are providing this early version of the manuscript. The manuscript will undergo copyediting, typesetting, and review of the resulting proof before it is published in its final form. Please note that during the production process errors may be discovered which could affect the content, and all legal disclaimers that apply to the journal pertain. 